\title{
Effect of Pharmacy Counseling on Readmissions in Patients with Acute Exacerbations of COPD: A Randomized Controlled Trial
}

\author{
Narachai Prasungriyo, B.Pharm., Nungruthai Sooksai, Ph.D. \\ Faculty of Pharmaceutical Sciences, Khon Kaen University, Mueang, Khon Kaen 40002, Thailand. \\ Received 12 October 2020 • Revised 1 December 2020 • Accepted 3 December 2020 • Published online 28 April 2021
}

\begin{abstract}
:
Objective: To investigate the effects of pharmacy counseling on clinical and economic outcomes in acute exacerbations of chronic obstructive pulmonary disease (AECOPD) patients.

Material and Methods: The outcomes consisted of 28-day hospital readmissions related to AECOPD, direct costs, medication adherence calculated by proportion of days covered (PDC), and health-related quality of life (HRQLL) measured by chronic obstructive pulmonary disease assessment test (CAT). The data derived from the intervention group, for which pharmacy counseling was provided, was compared with that obtained from the control group provided with usual pharmaceutical care. The study also drew comparisons between the PDC and CAT scores of pre- and postintervention periods.
\end{abstract}

Results: Forty-four patients (23 intervention and 21 control) were included in the analysis. There were no significant differences in the readmission rate ( $13 \%$ vs $19 \%$, p-value>0.050), nor the number of readmitted patients ( 3 vs 3 , p-value >0.050). A decrease in direct costs did not reach statistical significance ( $p$-value>0.050). In addition, no difference between the PDC scores was found (96.67 vs 100.00, p-value>0.050). Intervention patients obtained significantly lower CAT scores than the control patients did (9 vs 19, p-value<0.050). Compared with the pre-intervention period, PDC scores were identical; however, CAT scores measured during the post-intervention period were significantly different.

Conclusion: Pharmacy counseling for AECOPD patients could enhance HRQoL. Drug therapy and pulmonary rehabilitation may cause such improvement. Further work, which has adequate participants, is required to detect a significant difference in readmissions between the two groups.

Keywords: AECOPD, inpatients, pharmacy counseling, RCT, readmissions

Contact: Nungruthai Sooksai, Ph.D.

Faculty of Pharmaceutical Sciences, Khon Kaen University,

Mueang, Khon Kaen 40002, Thailand.

E-mail: nunsoo@kku.ac.th

(c) 2021 JHSMR. Hosting by Prince of Songkla University. All rights reserved.

This is an open access article under the CC BY-NC-ND license

(http://www.jhsmr.org/index.php/jhsmr/about/editorialPolicies\#openAccessPolicy).
J Health Sci Med Res 2021;39(6):467-479 doi: 10.31584 /jhsmr.2021806 www.jhsmr.org 


\section{Introduction}

It is acknowledged that chronic obstructive pulmonary disease (COPD) significantly affects the health of people all over the world. When the World Health Organization made a forward projection of diseases predicted to be the leading causes of death in 2030, COPD ranked third, after ischemic heart disease and cerebrovascular disease, respectively. ${ }^{1}$ As an economic issue, COPD places a large financial burden on the healthcare system. There is evidence that hospital admission rates related to exacerbations of COPD have a major influence on direct costs. ${ }^{2}$ Almost half of the healthcare costs for COPD patients correlate with hospitalizations. ${ }^{3}$ The increase in direct costs may directly relate to the frequency of COPD exacerbations and the severity of the disease. Similarly, the utilization of health care resources expressed as hospitalization rates aligns with the severity of COPD and its history of acute exacerbations of COPD (AECOPD). ${ }^{4}$

In addition to the economic impact, COPD can significantly affect health-related quality of life (HRQoL). ${ }^{5}$ There is significant impairment of HRQoL of COPD patients, no matter how their health status is. ${ }^{6}$ Compared to patients suffering from less than 3 exacerbations per year, patients suffering from more frequent exacerbations have a poorer HRQoL. ${ }^{7}$ The disease restrains patients from spending time with other people, and enjoying themselves in avocations. Addionally, by the same token, they appear frustrated with the lack of their ability to do what they want. ${ }^{5}$ In regard to disease management programs, many approaches have been developed to improve HRQoL in patients with COPD. To date; however, it is difficult to draw any firm conclusions for which intervention is the most efficacious in the enhancement of HRQoL. ${ }^{8}$

A large quantity of readmissions, due to COPD, increases the burden of the disease in addition to hospital admissions. ${ }^{9}$ The differences in hospital readmission rates differ by countries; for example, in Taiwan the rate is almost
$17.0 \%$, but this rate is even higher in other countries. The percentage of readmissions rises to $20.0 \%$ in London, to $22.0 \%$ in the United States, to $24.5 \%$ in Scotland and up to $25.0 \%$ in Australia. ${ }^{10,11}$

As a result of the excess of 30-day readmissions, COPD has been included in the Hospital Readmission Reduction Program in the United States since $2014 .^{12}$ Myers et al. ${ }^{13}$ analyzed the data of 904 hospitals to determine the effect of the implementation of such a program. Compared to the pre-implementation period, COPD readmissions during the post-implementation period significantly declined by $0.5 \%$ [95\% confident interval $(\mathrm{Cl}),-0.93$ to -0.12 , $\mathrm{p}$-value $=0.020]$. From this evidence, it is deduced that readmissions related to COPD may be preventable.

Much research in the field of pharmaceutical care has utilized pharmacist-led interventions for the purpose of decreasing the number of hospitalizations associated with COPD. Nevertheless, such studies have recruited only outpatients. ${ }^{14-16}$ As regards other work choosing hospitalized patients having a high chance of being readmitted to the hospital as study participants, no significant differences in hospital readmissions between the two groups have been evident in several studies. ${ }^{17-20}$ However, a reduction in the number of readmissions was found in the studies for which pre-discharge counseling and medication reconciliation were employed. ${ }^{21,22}$ It is concluded that patient care by a pharmacist could improve clinical outcomes. Although, readmission rates have been reduced significantly, because of pharmacy interventions, reducing readmissions and direct costs in hospitalized patients suffering from AECOPD has received scant attention in research into pharmaceutical care.

The electronic database provided by Wichianburi Hospital, in Thailand indicates that AECOPD ranked as one of the main causes of hospital admissions in 2016. Furthermore, it was recognized as the first- and sixthranking cause of hospital admissions in both male and 
female medical wards, respectively. Related to the economic aspect, this condition resulted in nearly 7 million Thai baht in direct costs. Research focusing on the issue of the financial ruin of the healthcare system has indicated that AECOPD was one of the three conditions that incurred financial losses; ${ }^{23}$ thus, to prevent further loss, it is important to prevent inpatients diagnosed with AECOPD from being re-hospitalized.

In light of the dramatic increase in the readmission rate, the primary objective of this randomized controlled trial was to reduce AECOPD-related readmissions, utilizing pharmacy counseling as the intervention. The secondary objectives were to reduce direct costs, maximize medication adherence, and improve HRQoL. This study not only compared these variables between the two groups but also analyzed the data on medication adherence and HRQoL collected during pre- and post-intervention periods.

\section{Material and Methods}

This study was a randomized, single-blind, controlled trial conducted at Wichianburi Hospital in Wichianburi district, Phetchabun province, between July 1, 2018 and December 31, 2019. Ethical approval for this study was obtained from the Institutional Review Board, Khon Kaen University (HE612189). A list of the COPD patients who had been admitted to the hospital with AECOPD in 2016 and 2017 was drawn from the hospital database, and randomization was performed afterwards by using SPSS software (version 23). Patients were blinded to the group to which they were assigned. Participants who were randomly allocated to the intervention group were afforded pharmacy counseling and usual pharmaceutical care, whereas those randomly allocated to the control group were provided only usual pharmaceutical care. The researcher offered incentives amounting to 200 Baht, so as to encourage patients in both groups to engage in the study. Additionally, participation in the study was expected to ease their transportation cost burdens. Each participant received a payment on the day of data collection. Data collection and the provision of pharmacy counseling were initiated when they were hospitalized for AECOPD during the study period.

Patients diagnosed with COPD were chosen to participate in the study if they were 20 years of age or over; they were admitted to either male or female medical wards or to the emergency room (ER) with AECOPD during the study period, had a history of AECOPD-related readmissions, had at least one medication history of COPD treatment, and were able to impart information about their medications, either by themselves or via their caregiver. Patients who were hospitalized for respiratory failure in the intensive care unit (ICU) or were unable to communicate in Thai were excluded from the study.

The estimated sample size was based on a piece of published data. ${ }^{21}$ The statistically significant difference between the two groups, in the number of readmitted patients, was drawn from the interventions that included pre-discharge pharmacist counseling. To decrease the readmission rate by approximately $70.0 \%$, it was determined that a sample size of 50 patients in each group needed to achieve a $5.0 \%$ significance level and $80.0 \%$ power.

All patients who granted consent were tagged as eligible subjects via a pop-up in the hospital program. Whenever they were admitted to the hospital or underwent AECOPD treatment at the ER, pharmacists would recognize them after entering their hospital number. The demographic and medication data were derived from the electronic medical record. For inpatients, the relevant information was gathered at their bedside, whereas for outpatients the information was collected at the counseling room.

For the primary outcomes, the number of hospital readmissions and readmitted patients was recorded, if AECOPD-related readmissions happened within 28 days of discharge. The secondary outcomes were comprised of direct costs, adherence scores, and HRQoL scores. Direct 
costs are those directly attributable to inpatient care, which included: nursing services, hospital bed services, drugs, medical supplies, laboratory investigation and pathology, and diagnostic imaging. Such costs would be analyzed if patients discharged from the hospital were readmitted due to AECOPD within 28 days. Data on medicationtaking behavior and HRQoL was collected at two different points. The participants' adherence was measured over a 90-day period before admission (pre-proportion of days covered scores) and after discharge (post-PDC scores). An inhaler, containing salmeterol and fluticasone; which is a combination of inhaled corticosteroids and long-acting beta2-agonists (ICS $\angle A B A$ ), was used as a proxy for assessing medication adherence. Patients were asked to complete a questionnaire about the impact of COPD once they became stable after undergoing medical treatment (pre-chronic obstructive pulmonary disease assessment test scores) and when they came to the hospital on followup visits scheduled in the next month following discharge (post-CAT scores).

The research pharmacist afforded intervention patients a one-time counseling session. The time spent on the counseling process was restricted to $30-45$ minutes. The content of the pharmacy counseling included the following: (1) basic knowledge of COPD, including pathophysiology and risk factors in the development of the disease, (2) the health consequences of smoking cigarettes, (3) a review of the technique for using inhalers, (4) the importance of medication adherence, (5) self-management and lifestyle modification, and (6) breathing exercises. To ensure that the patients retained the provided information the teachback or show-me technique was used. The patients were also encouraged to ask for further information after the counseling session. Furthermore, printed material containing succinct information regarding the disease, medications, and instructions on using the inhalers was made available to intervention patients. After the intervention patients were discharged, all were contacted by phone to investigate any adverse events and rectify any mistakes in taking of their medications. They were also informed that they should come to the hospital on their appointment date.

Together with medication dispensing, hospital pharmacists provided medication reconciliation at admission and discharge to both groups; this procedure was routinely performed for every patient.

$\mathrm{PDC}^{24}$ is one of the most well-known tools for assessing medication adherence based on dispensing data. The figure is calculated by dividing the total number of days' supply dispensed by the number of days during an observation period. The result is then multiplied by 100 , and the ratio is converted to a percentage. A threshold of $80.0 \%$ is generally referred to as optimal adherence. This study utilized an inhaler containing salmeterol and fluticasone as the medication for estimating participants' adherence level.

For this study, the COPD assessment test $(\mathrm{CAT})^{25}$ was used to explore the impact of COPD on health status. The assessment of cough, sputum, dyspnea, and chest tightness; collectively known as 'COPD symptoms', is performed globally using eight questionnaire items. This questionnaire uses a 5-point Likert scale to measure the extent to which these symptoms affect quality of life. CAT scores range from 0 to 40 , with higher scores indicating that patients were more severely affected by the disease. The CAT questionnaire was first administered when the patients either stayed in the hospital or visited the ER during the study period. For the completion of the second questionnaire, the patients had to attend the outpatient COPD clinic after discharge.

To compare the number of hospital readmissions within 28 days of discharge and the number of readmitted patients between the two groups, the chi-square test $\left(\mathrm{X}^{2}\right)$ was used. It was also utilized as statistics for analyzing 
gender and smoking status. The continuous parametric age of patients and the CAT scores, which were collected at their first visits and were normally distributed, were analyzed by the independent-samples t-test. Other variables, on which data were not normally distributed, were assessed by nonparametric statistics. The Mann-Whitney U-test was used for comparing the median of hospital readmissions, direct costs, the number of medications prescribed for the conditions of each patient prior to admission, peak expiratory flow rate (PEFR), CAT scores collected at the participants' second visits, and PDC scores. The PDC and CAT scores before and after providing the intervention (the pre- and post-intervention periods) were compared, and analyzed using the Wilcoxon signed-rank test: statistical significance was accepted at $p$-value $<0.050$.

\section{Results}

A retrospective search for eligible patients in the hospital database was conducted before the recruitment of participants. The results revealed that 222 patients had a history of hospitalization for AECOPD, between 2016 and 2017. COPD readmission; however, was found in 108 out of these. They were then randomly divided into two equal-sized groups: 54 participants were categorized as the intervention group, and the remaining participants were classified as the control group. Throughout the study period, individuals admitted to the hospital suffering from AECOPD accounted for $43.0 \%$ of patients giving their consent to the study (25 intervention and 21 control). Nevertheless, two cases in the intervention group were excluded from data analysis: one of them no longer took COPD medications; thereby being unable to be assessed for medication adherence, and the other was unable to be contacted for several months. Ultimately, 23 intervention and 21 control patients were included in the analysis (Figure 1).
The baseline characteristics of the intervention group were the same as those of the control group. Elderly men constituted nearly $100.0 \%$ of the patients in this study. Each group had only one patient who was an active smoker. There was a slight difference in PEFR between the two groups. More than half of the patients had other diseases in addition to COPD. The most common comorbid condition reported by the subjects was hypertension (43.1\%), followed by benign prostate hyperplasia (17.7\%) and dyslipidemia $(15.7 \%)$. The number of prescription medicines and the number of patients hospitalized after AECOPD earlier in the year were extremely similar in the intervention and control groups (Table 1).

This study intended primarily to decrease 28-day hospital readmissions among patients with AECOPD. During the study period, there were three patients in both groups re-hospitalized with AECOPD within 28 days of discharge; whereby, the data on hospital readmissions derived from them was included in the analysis. As shown in Table 2, no significant difference in the readmission rate between the two groups was evident; although, the number of readmissions in the intervention group was less than that of the control group ( $p$-value>0.050). Evidently, there was an equal number of readmitted patients in both groups. Therefore, Fisher exact test did not show a significant difference in the number of patients who were readmitted to the hospital ( $p$-value>0.050)

The control group had 21 more days of hospital stay than the intervention group. Although the length of stay in the hospital was noticeably different between these groups, the result was statistically insignificant ( $p$-value> 0.050). The comparison of direct costs of inpatient care was included in the secondary endpoints. Such costs, in the control group, were almost nine times as high as those in the intervention group. However, this result did not reach statistical significance. 


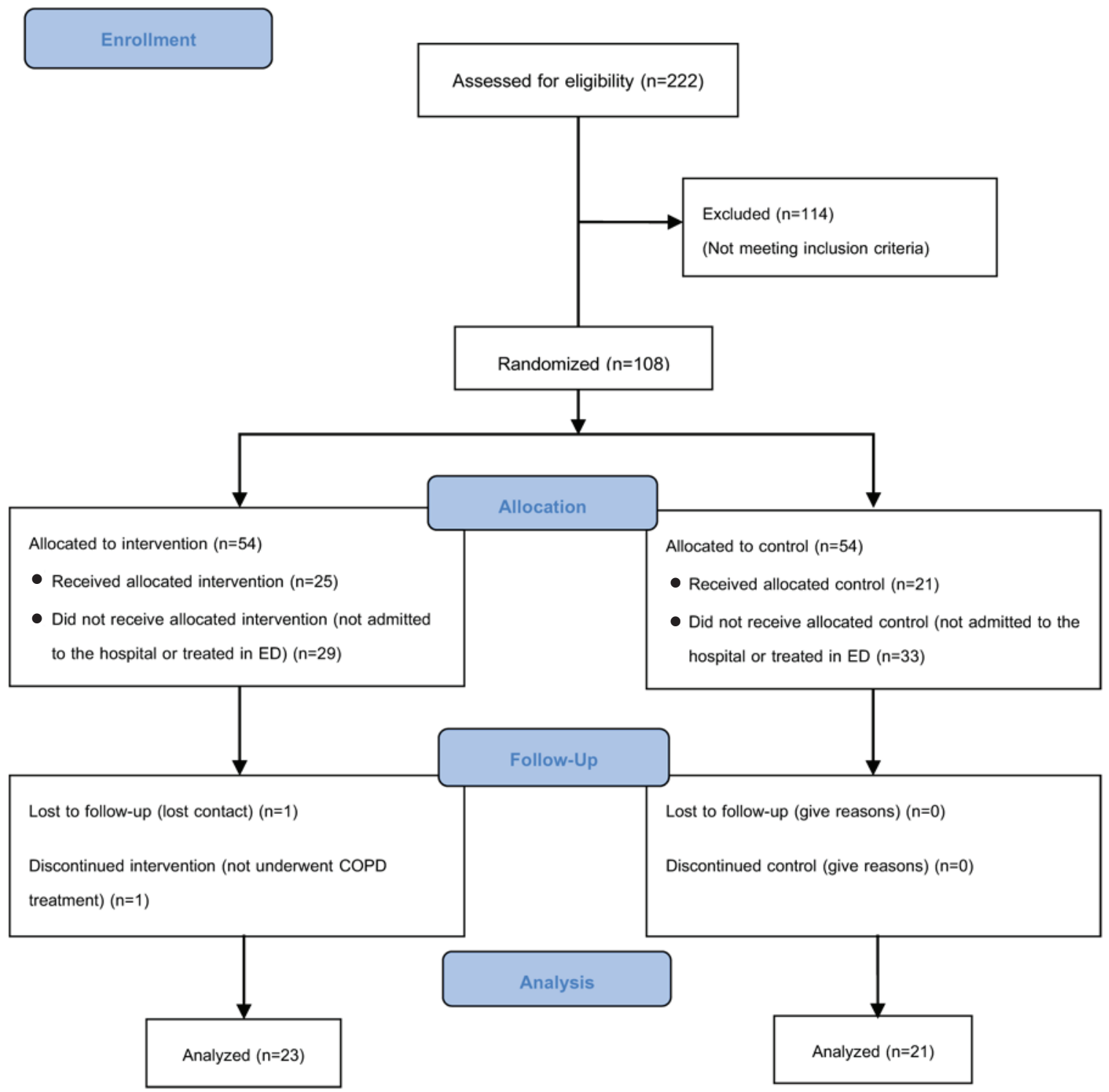

$\mathrm{ED}=$ emergency department, $\mathrm{COPD}=$ chronic obstructive pulmonary disease

Figure 1 Flow chart showing the number of patients in each stage of the study 
Table 1 Baseline characteristics of patients

\begin{tabular}{llll}
\hline Characteristics & $\begin{array}{l}\text { Control group } \\
(\mathbf{n = 2 1 )}\end{array}$ & $\begin{array}{l}\text { Intervention group } \\
(\mathbf{n = 2 3})\end{array}$ & \begin{tabular}{l}
$\mathbf{p}$-value \\
\hline Age (mean, S.D.)
\end{tabular} \\
Male (n, \%) & $69.65(7.901)$ & $69.14(10.489)$ & $0.856^{\star}$ \\
Smoking (n, \%) & $19(90.5)$ & $22(95.7)$ & $0.599^{\dagger}$ \\
PEFR (median, IQR) & $1(4.8)$ & $1(4.4)$ & $1.000^{\dagger}$ \\
The number of medications (median, IQR) & $250(200.00-300.00)$ & $300(230.00-350.00)$ & $0.193^{\ddagger}$ \\
Comorbidities (n, \%) & $6.0(6.00-8.00)$ & $6.0(5.00-8.00)$ & $0.801^{\ddagger}$ \\
One-year previous hospitalizations (n, \%) & $16(76.2)$ & $15(65.2)$ & $0.426^{\dagger}$ \\
\hline
\end{tabular}

*Independent-samples t-test, $† \mathrm{X}^{2}$ test, $\ddagger$ Mann-Whitney U-test

S.D.=standard deviation, PEFR=peak expiratory flow rate, $I Q R=$ interquartile range

Table 2 Comparison of hospital readmissions, readmitted patients, direct costs, pre-PDC and CAT scores, and post-PDC and CAT scores

\begin{tabular}{llc} 
Variables & $\begin{array}{l}\text { Control group } \\
(\mathbf{n = 2 1})\end{array}$ & $\begin{array}{l}\text { Intervention group } \\
(\mathbf{n = 2 3})\end{array}$ \\
\hline Hospital readmissions (n, \%) & $4(19.0)$ & $3(13.0)$ \\
Readmitted patients (n) & 3 & 3 \\
Direct costs (Baht) & 206,827 & 23,640 \\
Pre-PDC scores (median, IQR) & $100.00(98.36-100.00)$ & $100.00(71.11-100.00)$ \\
Post-PDC scores (median, IQR) & $0.000^{\star}$ \\
Pre-CAT scores (mean, S.D.) & $96.67(67.78-100.00)$ & $100.00(88.89-100.00)$ \\
Post-CAT scores (median, IQR) & $14.24(6.33)$ & $0.217^{\dagger}$ \\
\hline
\end{tabular}

† Mann-Whitney U-test, *Fisher Exact test, ‡ Independent-samples t-test

$\mathrm{PDC}=$ proportion of days covered, IQR=interquartile range, CAT=COPD assessment test, S.D.=standard deviation

Further details about the length of stay and direct costs of each readmitted patients were demonstrated in Table 3. The results showed that the second and third control patients had a hospital stay for over 10 days. One of those was admitted to an ICU, due to AECOPD with respiratory failure for 10 days. They were then transferred to the male medical ward (total 16 days). The other was readmitted to the female medical ward two times. Hence, the control group had much higher direct costs of inpatient care. For the intervention group, patients were readmitted to the male medical ward only.
At baseline, participants who adhered to the medication accounted for $73.9 \%$ of all intervention patients. Likewise, over two-thirds of the control patients had high adherence scores (85.7\%). The Mann-Whitney U-test revealed that the pre-PDC scores of the two groups were not significantly different ( $p-v a l u e>0.050)$. Correspondingly, no evidence was found for a significant difference in the post-PDC scores ( $p$-value>0.050).

The independent-samples t-test was used to compare the mean of the pre-CAT scores, due to the normal data distribution. The t-test demonstrated that 
Table 3 Comparison between number of readmissions, length of stay, and direct costs

\begin{tabular}{|c|c|c|c|c|c|c|}
\hline \multirow{2}{*}{ Readmitted patients } & \multicolumn{3}{|c|}{ Control group } & \multicolumn{3}{|c|}{ Intervention group } \\
\hline & $\begin{array}{l}\text { Readmissions } \\
\text { (n) }\end{array}$ & $\begin{array}{l}\text { LOS } \\
\text { (days) }\end{array}$ & $\begin{array}{l}\text { Costs } \\
\text { (Baht) }\end{array}$ & $\begin{array}{l}\text { Readmissions } \\
\text { (n) }\end{array}$ & $\begin{array}{l}\text { LOS } \\
\text { (days) }\end{array}$ & $\begin{array}{l}\text { Costs } \\
\text { (Baht) }\end{array}$ \\
\hline 1 & 1 & 4 & 6,598 & 1 & 3 & 3,884 \\
\hline 2 & 1 & 16 & 180,538 & 1 & 6 & 12,312 \\
\hline 3 & 2 & 13 & 19,691 & 1 & 3 & 7,444 \\
\hline Total & 4 & 33 & 206,827 & 3 & 12 & 23,640 \\
\hline
\end{tabular}

LOS=length of stay

Table 4 Comparison between pre- and post-intervention period

\begin{tabular}{|c|c|c|c|}
\hline Variables & Pre-intervention & Post-intervention & p-value \\
\hline PDC scores (median, IQR) & $100(71.11-100.00)$ & $100(88.89-100.00)$ & $0.133^{*}$ \\
\hline CAT scores (median, IQR) & $13(10.00-23.00)$ & $9.00(3.00-12.00)$ & $0.000^{*}$ \\
\hline
\end{tabular}

*Wilcoxon signed-rank test

$\mathrm{PDC}=$ proportion of days covered, CAT=COPD assessment test, IQR=interquartile range

the two groups had comparable scores ( $p$-value>0.050). However, there was a significant difference between the two groups in the post-CAT scores; using the Mann-Whitney U-test ( $p$-value<0.050).

Not only were comparisons made between the two groups to determine whether pharmacy counseling could improve clinical and economic outcomes, but also this present study compared the PDC and CAT scores of the intervention group during the pre- and post-intervention periods. The results showed that there was no statistically significant difference in PDC scores ( $p$-value>0.050; Table 4). Regarding the other outcome, a substantial decline was noted in CAT scores measured in the post-intervention period ( $p$-value<0.050; Figure 2).

\section{Discussion}

The findings derived from this study both rejected and corroborated the hypotheses. It was found that pharmacy counseling failed to resolve the problem of excessive readmissions. Notwithstanding, it could raise HRQoL.

This study was designed to address the issue of hospital readmissions in patients suffering from COPD exacerbations, by the utilization of pharmacy counseling at bedside, which was not integrated into pharmaceutical care practices in the setting. After completing data analysis, this present study did not detect any evidence for reductions in the readmission rate nor the number of patients readmitted to the hospital. This might be attributed to a small sample size, which is recognized to be the main problem of the study. This result is in agreement with that of prior work. Laswell et al. ${ }^{20}$ conducted a pilot study, with the purpose of reducing readmissions and emergency department visits in COPD patients hospitalized after COPD attacks. The intervention group underwent patient counseling before discharge; whereas, matched retrospective patients referred to as the comparison group did not received the intervention. 


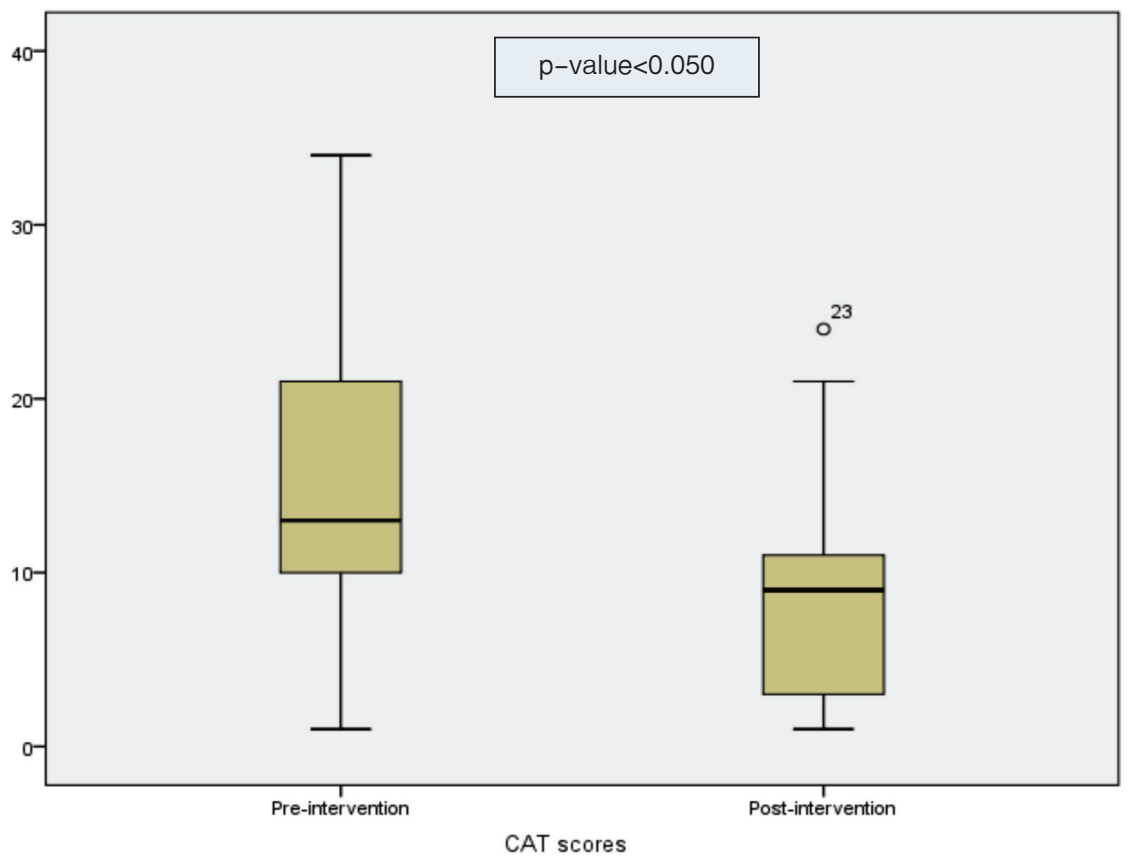

Figure 2 Box plot of a comparison between pre-and post-intervention median scores of the intervention group, derived from the CAT questionnaire. The p-value displayed at the top of the figure denotes a statistically significant difference using the Wilcoxon signed-rank test. An outlier shows that the value of Case 23 is beyond 1.5 times IQR.

They found that the rate of 30- and 90-day readmissions was statistically insignificant $(p-$ value $=1.000$ and 0.589 , respectively).

Many approaches have been applied to reduce healthcare utilization. Prieto-Centurion et al. ${ }^{26}$ conducted a review of research into interventions for reducing hospital readmissions due to AECOPD. A multicenter, randomized, controlled trial by Bourbeau et al. ${ }^{27}$, which was one of the studies selected for the review, launched a program designed to educate COPD patients about self-management. The study revealed that there was a $35.0 \%$ decrease in the number of patients hospitalized after suffering from AECOPD during a follow-up period. This result implies that a self-management program would be an important element of patient counseling. This present study; therefore, provided concise recommendations on how to manage the symptoms of a COPD exacerbation.

Previous studies have noted that pharmacist-led interventions have a positive effect on hospital admissions. Hence, providing a counseling program for COPD outpatients could lower hospital admissions significantly. ${ }^{14,16,28}$ In an investigation into medication reconciliation for and the counseling of older adult inpatients before hospital discharge, the result demonstrated that the number of readmitted patients was considerably decreased in comparison with the control group. ${ }^{21}$

In the literature, very little was found on the effect of pharmacist interventions on the direct costs of inpatient care. In a systematic review of studies on inpatient care by clinical pharmacists, the research on pharmacist 
involvement in ward rounds demonstrates a significant decline in hospital charges. ${ }^{29}$ In the same way, a study by Gallagher et al. ${ }^{30}$ demonstrated that interventions conducted by pharmacists have a beneficial effect on cost avoidance.

In the outcome of this study, pharmacy counseling dramatically reduced direct costs related to AECOPD, although the number of hospital readmissions in the two groups remained equal. The excessive direct costs of the control group were largely due to the fact that the patient was admitted to the intensive care unit and the internal medical ward for almost a month. This yield contradicts that of another study in which medication reconciliation was utilized. The average cost of hospital readmissions attributed to respiratory causes increased slightly when it was compared with the baseline. ${ }^{22}$ Therefore, it is evident that pharmacy counseling could be an intervention that when implemented reduces healthcare utilization; although no significant reduction in such costs was found when compared with the control group.

Medication adherence.

In real-world settings, COPD patients exhibit suboptimal adherence to inhaled medications, which is contrary to the findings of this study. Montes de Oca et al. ${ }^{31}$ performed an observational, cross-sectional, multinational, multicenter study with the purpose of investigating the level of patient adherence. The percentage of patients presenting good adherence, assessed by the test of adherence to inhalers, was $54.1 \%$, and when assessed by the 8 -item Morisky Medication Adherence Scale (MMAS-8), was $51.0 \%$. Agh et al. ${ }^{32}$ conducted an observational, crosssectional study in which MMAS was employed to analyze the adherence pattern. They found that nearly $60.0 \%$ of patients were adherent to COPD medications. These results are consistent with the data derived from a systematic review of the research into adherence to COPD treatment. The findings from the evaluation of adherence based on the test of adherence to inhalers and MMAS-8 showed that adherent patients accounted for less than $50.0 \%$ of subjects. $^{33}$

Regarding adherence measures, PDC has been generally employed to analyzed prescription dispensing databases. ${ }^{24}$ This study involved this method to calculate medication adherence scores. The denominator of the calculation was three months in all cases. On the contrary, such a period of time differed from that of other work. Zhang et al. $^{34}$ who did a calculation of PDC using 180 days as the follow-up period. Wu et al. ${ }^{35}$ calculated PDC based on the number of days medications dispensed for 30 and 180 days. Bogart et al. ${ }^{36}$ performed an adherence calculation, for which 365 days were defined as the study period. Similarly, Cyr et al. ${ }^{37}$ and Barrecheguren et al. ${ }^{38}$ used the same interval for computing PDC. The results of the studies in which a 365-day period is used for the PDC calculation confirm that patients with COPD have low adherence to drug therapy. As a result, the duration for the PDC calculation should be extended to 365 days to identify the difference in adherence scores between the two groups. Moreover, although medication-taking behavior can be measured by several approaches, there are no ones that can be the gold standard. The appropriate adherence measures depend on the characteristics of patients and medications, the needs of clinicians and the purpose of each assessment tool. Choosing more than one measure could make the result be more accurate.

Despite poor medication adherence among COPD patients in real-life events as mentioned above, this present study demonstrated that the difference in adherence scores between the two groups was statistically insignificant. However, it is assumed that pharmacy counseling might enhance medication-taking behavior as the number of intervention patients who were adherent to inhaled corticosteroids and long-acting beta2-agonists (ICS $/ \mathrm{ABA})$ was 1.4 times more than the control patients. 
In the present study, a comparison of the CAT scores of the intervention group with those of the control group showed an obvious difference in HRQoL between the two groups. Such a result suggests that pharmacy counseling may reduce the impact of COPD on patient health status. This agrees with earlier observations, in that there is a significant improvement in CAT scores after the implementation of integrated disease management ${ }^{39}$; another study by Khachi et al. $^{40}$ also revealed the same result.

Furthermore, these findings corroborate the results of the previous work, in which the St. George's Respiratory Questionnaire (SGRQ) was utilized as an assessment of HRQoL. Khdour et al. ${ }^{14}$ conducted a study on the effects of a disease and medicine management program performed by a clinical pharmacist on the improvement of quality of patients' lives. They reported that the SGRQ scores of intervention patients significantly improved compared to those of control patients. The result of the study corresponds with that of the study by Wei et al. ${ }^{16}$ The authors noticed that the intervention group exhibited a marked difference in the two subscales-symptom and impact-of the SGRQ questionnaire, compared to that of the control group.

A study of the events at two points in time was conducted to confirm whether the intervention improved the medication adherence and health status of COPD patients. The findings are consistent with the results derived from the comparison between the intervention and the control groups. It is possible that pharmacy counseling could be an effective intervention for improving HRQoL for patients who have been hospitalized after AECOPD. Certain factors, such as medical care being provided by a physician and pulmonary rehabilitation being performed by a physical therapist, may have influenced the improvement of the patients' health.

The difference in hospital readmissions, which was undetected, may have been due to a small sample size. This problem was regarded as the major limitation of this study. Future research, having substantial participants and being designed as a multi-center trial, should be undertaken to increase generalizability of the findings and detect a statistically significant difference in readmissions between the two groups.

All cases in the intervention group were provided with pharmacy counseling by the research pharmacist only one time. Nevertheless, there is a tendency for the effectiveness of the pharmacist intervention to decrease as time passes. ${ }^{14}$ Thus, it is important to provide the intervention and follow up patients more than once. As regard to the primary outcome measure time frame, a 28-day duration may be insufficient to convincingly demonstrate the effect of pharmacy counseling upon hospital readmission reduction.

\section{Conclusion}

This study was undertaken to cope with excessive AECOPD readmission rates. Although, pharmacy counseling was unsuccessful in reducing readmissions, which was the primary outcome, the intervention showed a dramatic improvement in HRQoL. Furthermore, this study revealed a reduction in direct costs, which was attributed to a decline in the length of hospital stay. Consequently, the incorporation of pharmacists into standard inpatient care would be a useful approach in mitigating the effects of COPD exacerbation on both clinical and economic outcomes.

\section{Acknowledgement}

I would like to express my profound gratitude to my supervisor for her professional guidance and useful critique of this work. I would also like to thank the multidisciplinary team and my colleagues for facilitating the data collection.

\section{Funding sources}

This study was partially funded by the Faculty of Pharmaceutical Sciences, Khon Kaen University. The 
source of funding had no effect on the identification of a research problem, nor the selection of journals for the publication of this study.

\section{Conflict of interest}

There are no potential conflicts of interest to declare.

\section{References}

1. Parris BA, O’Farrell HE, Fong KM, Yang IA. Chronic obstructive pulmonary disease (COPD) and lung cancer: common pathways for pathogenesis. J Thorac Dis 2019;11(Suppl 17):S215572.

2. May SM, Li JTC. Burden of chronic obstructive pulmonary disease: healthcare costs and beyond. Allergy Asthma Proc 2015;36:4-10.

3. Chapman KR, Mannino DM, Soriano JB, Vermeire PA, Buist AS, Thun MJ, et al. Epidemiology and costs of chronic obstructive pulmonary disease. Eur Respir J 2006;27:188-207.

4. Iheanacho I, Zhang S, King D, Rizzo M, Ismaila AS. Economic burden of chronic obstructive pulmonary disease (COPD): a systematic literature review. Int $\mathrm{J}$ Chron Obstruct Pulmon Dis 2020;15:439-60.

5. Zamzam MA, Azab NY, El Wahsh RA, Ragab AZ, Allam EM. Quality of life in COPD patients. Egypt $J$ Chest Dis Tuberc 2012;61:281-9.

6. King D, Iheanacho I, Zhang S, Kenny J, Rizzo M, Ismaila A. PRS101 - Impact of chronic obstructive pulmonary disease (COPD) on quality of life: findings from a systematic literature review. Value Heal 2018;21:S420-1.

7. Hurst JR, Skolnik N, Hansen GJ, Anzueto A, Donaldson GC, Dransfield MT, et al. Understanding the impact of chronic obstructive pulmonary disease exacerbations on patient health and quality of life. Eur J Intern Med 2020;73:1-6.

8. Niesink A, Trappenburg JCA, de Weert-van Oene GH, Lammers JWJ, Verheij TJM, Schrijvers AJP. Systematic review of the effects of chronic disease management on quality-of-life in people with chronic obstructive pulmonary disease. Respir Med 2007;101:2233-9.

9. Tang FW, Lee DT. A phenomenological study of hospital readmissions of Chinese older people with COPD. Gerontologist 2017;57:1113-22.
10. Shah T, Press VG, Huisingh-Scheetz M, White SR. COPD readmissions: addressing COPD in the era of value-based health care. Chest 2016;150:916-26.

11. Hakim MA, Garden FL, Jennings MD, Dobler CC. Performance of the LACE index to predict 30-day hospital readmissions in patients with chronic obstructive pulmonary disease. Clin Epidemiol 2017;10:51-9.

12. Braman SS. Hospital readmissions for COPD: we can meet the challenge. Chronic Obstr Pulm Dis 2015;2:4-7.

13. Myers LC, Faridi MK, Hasegawa K, Hanania NA, Camargo CA. The hospital readmissions reduction program and readmissions for chronic obstructive pulmonary disease, 20062015. Ann Am Thorac Soc 2019;17:450-6.

14. Khdour MR, Kidney JC, Smyth BM, McElnay JC. Clinical pharmacy-led disease and medicine management programme for patients with COPD. Br J Clin Pharmacol 2009;68:588-98.

15. Jarab AS, Alqudah SG, Khdour M, Shamssain M, Mukattash TL. Impact of pharmaceutical care on health outcomes in patients with COPD. Int J Clin Pharm 2012;34:53-62.

16. Wei L, Yang X, Li J, Liu L, Luo H, Zheng Z, et al. Effect of pharmaceutical care on medication adherence and hospital admission in patients with chronic obstructive pulmonary disease (COPD): a randomized controlled study. J Thorac Dis 2014;6:656-62.

17. Nazareth I, Burton A, Shulman S, Smith P, Haines A, Timberall H. A pharmacy discharge plan for hospitalized elderly patients - A randomized controlled trial. Age Ageing 2001;30: 33-40.

18. Stowasser DA, Collins DM, Stowasser M. A randomised controlled trial of medication liaison services - patient outcomes. J Pharm Pract Res 2002;32:133-40.

19. Schnipper JL, Kirwin JL, Cotugno MC, Wahlstrom SA, Brown BA, Tarvin E, et al. Role of pharmacist counseling in preventing adverse drug events after hospitalization. Arch Intern Med 2006;166:565-71.

20. Laswell E, Svelund E, Harzler M, Chambers K, Chen A. Discharge medication counseling and its correlation with reducing readmissions for patients with chronic obstructive pulmonary disease exacerbations. Inov Pharm 2015;6:1-8.

21. Al-Rashed SA, Wright DJ, Roebuck N, Sunter W, Chrystyn H. The value of inpatient pharmaceutical counselling to elderly patients prior to discharge. Br J Clin Pharmacol 2002;54:65764. 
22. Eisenhower C. Impact of pharmacist-conducted medication reconciliation at discharge on readmissions of elderly patients with COPD. Ann Pharmacother 2013;48:203-8.

23. Phakdiphan S, Anantachoti P, Pengsuparp T, Trakunkan S. Hospital financial status and quality of care under diagnosis related groups payment machanism. Thai Pharm Heal Sci J 2014;9:213-21.

24. Raebel MA, Schmittdiel J, Karter AJ, Konieczny JL, Steiner JF. Standardizing terminology and definitions of medication adherence and persistence in research employing electronic databases. Med Care 2013;51(8 Suppl 3):S11-21.

25. Jones PW, Harding G, Berry P, Wiklund I, Chen WH, Kline Leidy N. Development and first validation of the COPD Assessment Test. Eur Respir J 2009;34:648-54.

26. Prieto-Centurion V, Markos MA, Ramey NI, Gussin HA, Nyenhuis $\mathrm{SM}$, Joo MJ, et al. Interventions to reduce rehospitalizations after chronic obstructive pulmonary disease exacerbations. A systematic review. Ann Am Thorac Soc 2014;11:417-24.

27. Bourbeau J, Julien M, Maltais F, Rouleau M, Beaupré A, Bégin $R$, et al. Reduction of hospital utilization in patients with chronic obstructive pulmonary disease: a disease-specific selfmanagement intervention. Arch Intern Med 2003;163:585-91.

28. Tommelein E, Mehuys E, Van Hees T, Adriaens E, Van Bortel L, Christiaens T, et al. Effectiveness of pharmaceutical care for patients with chronic obstructive pulmonary disease (PHARMACOP): a randomized controlled trial. Br J Clin Pharmaco 2014;77:756-66

29. Kaboli PJ, Hoth AB, McClimon BJ, Schnipper JL. Clinical pharmacists and inpatient medical care: a systematic review. Arch Intern Med 2006;166:955-64.

30. Gallagher J, Byrne S, Woods N, Lynch D, McCarthy S. Costoutcome description of clinical pharmacist interventions in a university teaching hospital. BMC Health Serv Res 2014;14:177.

31. Montes de Oca M, Menezes A, Wehrmeister FC, Lopez Varela MV, Casas A, Ugalde L, et al. Adherence to inhaled therapies of COPD patients from seven Latin American countries: The
LASSYC study. PLoS One 2017;12. doi: 10.1371/journal.pone. 0186777.

32. Agh T, Inotai A, Meszaros A. Factors associated with medication adherence in patients with chronic obstructive pulmonary disease. Respiration 2011;82:328-34.

33. Świątoniowska-Lonc N, Chabowski M, Polański J, Mazur G, Jankowska-Polańska B. Adherence to therapy in chronic obstructive pulmonary disease: a systematic review. Adv Exp Med Biol 2020;1271:37-47.

34. Zhang S, Thomas. Adherence to chronic obstructive pulmonary disease maintenance medication among patients with copd. Value Heal 2015;18:A177-8.

35. Wu AC, Butler MG, Li L, Fung V, Kharbanda EO, Larkin EK, et al. Primary adherence to controller medications for asthma is poor. Ann Am Thorac Soc 2015;12:161-6.

36. Bogart M, Stanford RH, Laliberté F, Germain G, Wu JW, Duh MS. Medication adherence and persistence in chronic obstructive pulmonary disease patients receiving triple therapy in a USA commercially insured population. Int J COPD 2019; 14:343-52.

37. Cyr MC, Beauchesne MF, Lemière C, Blais L. Comparison of the adherence and persistence to inhaled corticosteroids among adult patients with public and private drug insurance plans. J Popul Ther Clin Pharmacol 2013;20:26-41.

38. Barrecheguren M, Monteagudo M, Miravitlles M. Populationbased study of LAMA monotherapy effectiveness compared with LABA $L A M A$ as initial treatment for COPD in primary care. npj Prim Care Respir Med 2018;28. doi: 10.1038/s41533018-0102-x.

39. Ferrone M, Masciantonio MG, Malus N, Stitt L, O’Callahan T, Roberts Z, et al. The impact of integrated disease management in high-risk COPD patients in primary care. npj Prim Care Respir Med 2019;29. doi: 10.1038/s41533-019-0119-9.

40. Khachi $\mathrm{H}$, Karikari P. P78 impact of a pharmacist-led asthma and COPD reviews in general practice. Thorax 2013;68 (Suppl 3):A110-1. 